Mathematical Modelling And AnAlysis

Volume 16 Number 3, September 2011, 365-375

Doi:10.3846/13926292.2011.601768

(c) Vilnius Gediminas Technical University, 2011
Publisher: Taylor\&Francis and VGTU

www.tandfonline.com/loi/tmma20

Online ISSN: $1648-3510$

Print ISSN: 1392-6292

\title{
Oscillation Analysis of Numerical Solutions for Nonlinear Delay Differential Equations of Population Dynamics*
}

\author{
Jianfang $\mathrm{Gao}^{a}$, Minghui Song ${ }^{b}$ and Mingzhu $\mathrm{Liu}^{b}$ \\ ${ }^{a}$ School of Mathematical Sciences, Harbin Normal University \\ Harbin, 150025, China \\ ${ }^{b}$ Department of mathematics, Harbin Institute of Technology \\ Harbin, 150001, China \\ E-mail(corresp.): 09151108@163.com \\ E-mail: minghuisong@hit.edu.cn; mzliu@hit.edu.cn
}

Received November 10, 2009; revised May 13, 2011; published online August 1, 2011

\begin{abstract}
This paper is concerned with oscillations of numerical solutions for the nonlinear delay differential equation of population dynamics. The equation proposed by Mackey and Glass for a "dynamic disease" involves respiratory disorders and its solution resembles the envelope of lung ventilation for pathological breathing, called Cheyne-Stokes respiration. Some conditions under which the numerical solution is oscillatory are obtained. The properties of non-oscillatory numerical solutions are investigated. To verify our results, we give numerical experiments.
\end{abstract}

Keywords: Oscillation, nonlinear, delay differential equations, numerical methods, population dynamics.

AMS Subject Classification: 34M10.

\section{Introduction}

Recently, there have been a lot of studies concerning the oscillatory behaviour of delay differential equations and difference equations. In particular, the literature on the applications of oscillation theory for nonlinear delay differential equations and difference equations is growing rapidly $[4,7,11,12,14,16]$. This study is very interesting in applications in some mathematical models, such as ecology, biology, spread of some infectious diseases in humans and so on. But to the best of our knowledge, only a few papers have been devoted to oscillations of numerical solutions for delay differential equations(see $[8,9])$. The papers [8]

\footnotetext{
* This work is supported by the Educational Department Scientific Technology Program of Heilongjiang Province (11551136) and Academic Foundation for Youth of Harbin Normal University (KJB200816).
} 
and [9] are concerned with oscillations of numerical solutions for linear delay differential equations.

In this paper, oscillations of numerical solutions for the nonlinear delay differential equations of population dynamics is studied. We consider the equation

$$
x^{\prime}(t)+\frac{\alpha V_{m} x(t) x^{p}(t-\tau)}{\beta^{p}+x^{p}(t-\tau)}=\lambda, \quad t \geq 0,
$$

with conditions

$$
\alpha, V_{m}, \beta, \tau, \lambda, p \in(0, \infty) .
$$

Equation (1.1) is proposed by Mackey and Glass [10] for a "dynamic disease" which involves respiratory disorders, where $x(t)$ denotes the arterial $\mathrm{CO}_{2}$ concentration of a mammal, $\lambda$ is the $\mathrm{CO}_{2}$ production rate, $V_{m}$ denotes the maximum ventilation rate, and $\tau$ is the time between oxygenation of blood in lungs and stimulation of chemoreceptors in the brainstem. The solution of Eq.(1.1) resembles the envelope of lung ventilation for pathological breathing, called Cheyne-Stokes respiration. It is known that such respiration is observed in the conditions of severe, life threatening pathology, when for some reason the duration of travel of blood from lungs to the chemoreceptors located in the brain stem increases or, as a result of the deterioration of some neurons in the brain stem, sensitivity of the ventilation to the $\mathrm{CO}_{2}$ concentration rises. Cheyne-Stokes respiration is also observed in healthy subjects in specific conditions, e.g., high in mountains where oxygen is insufficient. In this paper, we will investigate some sufficient conditions under which the numerical solution is oscillatory. We also investigate the behaviour of non-oscillatory numerical solutions.

As usual, a function $x(t)$ is said to be oscillatory about $K$, if the function $x(t)-K$ has arbitrarily large zeros. We say that a function $x(t)$ oscillates if it oscillates about zero.

Similarly, a sequence $\left\{x_{n}\right\}$ is said to oscillate about $\left\{y_{n}\right\}$ if $\left\{x_{n}-y_{n}\right\}$ is neither eventually positive nor eventually negative. If $\left\{y_{n}\right\}=\{\mathrm{y}\}$ is a constant sequence, we simply say that $\left\{x_{n}\right\}$ oscillates about y. In particular, when $\left\{y_{n}\right\}$ $=\{0\}$, we just say that $\left\{x_{n}\right\}$ oscillates.

Definition 1. We say a differential equation is oscillatory if all the solutions of the equation are oscillatory.

We consider the solution of equation (1.1) with the initial condition of the form

$$
x(t)=\varphi(t), \quad-\tau \leq t \leq 0, \quad \varphi \in C([-\tau, 0],(0, \infty)) .
$$

In view of [5], we know that Eq. (1.1) can be reduced to

$$
y^{\prime}(t)=y(t)\left[\frac{p_{1}}{q_{1}+y^{p}(t-\tau)}-\lambda y(t)\right]
$$

by setting $x(t)=1 / y(t)$. Let $y(t)=k e^{z(t)}$,we can get

$$
z^{\prime}(t)+\lambda k f_{1}(z(t))+M f_{2}(z(t-\tau))=0,
$$


where

$$
f_{1}(u)=e^{u}-1, \quad f_{2}(u)=\frac{q_{1}+k^{p}}{p} \frac{e^{p u}-1}{q_{1}+k^{p} e^{p u}},
$$

$M=p_{1} p k^{p} /\left(q_{1}+k^{p}\right)^{2}, p_{1}=\alpha V_{m} / \beta^{p}, q_{1}=1 / \beta^{p}$ and $k$ is the positive equilibrium point of (1.4). Then $K^{*}=1 / k$ is the equilibrium point of (1.1) and $x(t)$ oscillates about $K^{*}$ if and only if $z(t)$ oscillates.

The following theorems are helpful in presenting the main results of the paper.

Theorem 1 [[5]]. Assume that (1.2) holds and $M=p_{1} p k^{p} /\left(q_{1}+k^{p}\right)^{2}, p_{1}=$ $\alpha V_{m} / \beta^{p}, q_{1}=1 / \beta^{p}$, then every positive solution of (1.1) oscillates about the equilibrium point $K^{*}$ if $M e^{\lambda k \tau} \tau>1 / e$.

Theorem 2 [[2]]. Consider the difference equation

$$
a_{n+1}-a_{n}+\Sigma_{j=-k}^{l} q_{j} a_{n+j}=0
$$

Assume that $k, l \in \mathbb{N}$ and $q_{j} \in \mathbb{R}$ for $j=-k, \cdots, l$. Then the following statements are equivalent.

- Every solution of equation (1.6) oscillates.

- The characteristic equation $\lambda-1+\Sigma_{j=-k}^{l} q_{j} \lambda^{j}=0$ has no positive roots.

Theorem 3 [see [2]]. Consider the difference equation

$$
a_{n+1}-a_{n}+p a_{n-k}+q a_{n}=0,
$$

where $k>0, p>0$ and $q>0$. Then the necessary and sufficient conditions for the oscillation of all solutions of $(1.7)$ are $q \in(0,1)$ and $p K>(1-q)^{k+1}$, where $K$ denotes the constant $(k+1)^{k+1} / k^{k}$.

The following lemma is obvious.

Lemma 1. For $x>-1$ and $x \neq 0$, we have $\ln (1+x)>x /(1+x)$.

\section{Oscillations of Numerical Solutions}

Applying the linear $\theta$-method to equation (1.5), we get

$$
\begin{aligned}
z_{n+1}= & z_{n}-h \theta \lambda k f_{1}\left(z_{n+1}\right)-h \theta M f_{2}\left(z_{n+1-m}\right) \\
& -h(1-\theta) \lambda k f_{1}\left(z_{n}\right)-h(1-\theta) M f_{2}\left(z_{n-m}\right),
\end{aligned}
$$

where $0 \leq \theta \leq 1, h=\tau / m, m$ is a positive integer. $z_{n+1}$ and $z_{n+1-m}$ are approximations to the analytic solutions $z(t)$ and $z(t-\tau)$ of $(1.5)$ at $t_{n+1}$. Let $z_{n}=-\ln \left(k x_{n}\right)$, then

$$
\begin{aligned}
x_{n+1}= & x_{n} \exp \left(\frac{h \theta \lambda}{x_{n+1}}+\frac{h(1-\theta) \lambda}{x_{n}}-h \lambda k+h \theta \lambda k \beta^{p} \frac{1-k^{p} x_{n+1-m}^{p}}{\beta^{p}+x_{n+1-m}^{p}}\right. \\
& \left.+h(1-\theta) \lambda k \beta^{p} \frac{1-k^{p} x_{n-m}^{p}}{\beta^{p}+x_{n-m}^{p}}\right)
\end{aligned}
$$


Definition 2. We call the formula (2.2) as the exponential linear $\theta$-method for (1.1), where $0 \leq \theta \leq 1, h=\tau / m, m$ is a positive integer, $x_{n+1}$ and $x_{n+1-m}$ are approximations to the analytic solutions $x(t)$ and $x(t-\tau)$ of (1.1) at $t_{n+1}$.

Theorem 4. The numerical method (2.2) is convergent. Further, it is of the first order when $\theta \neq 1 / 2$ and of the second order when $\theta=1 / 2$.

Proof. In view of [3], we can solve the equation (1.5) by the method of steps. In the following, we introduce it in brief. We have the following initial value problem from (1.3)

$$
\begin{aligned}
& z^{\prime}(t)+\lambda k f_{1}(z(t))+M f_{2}(z(t-\tau))=0, t>0, \\
& z(t)=-\ln (k \varphi(t)),-\tau \leq t \leq 0, \quad \varphi \in C[[-\tau, 0],(0, \infty)] .
\end{aligned}
$$

Hence equation (1.5) becomes

$$
z^{\prime}(t)+\lambda k f_{1}(z(t))+M f_{2}(-\ln (k \varphi(t-\tau)))=0
$$

for $t \in[0, \tau]$. We denote $z(t)$ as $z_{1}(t)$ for $t \in[0, \tau]$.

When $t \in[\tau, 2 \tau]$, equation (1.5) becomes

$$
\left.z^{\prime}(t)+\lambda k f_{1}(z(t))+M f_{2}\left(z_{1}(t-\tau)\right)\right)=0 .
$$

We denote $z(t)$ as $z_{2}(t)$ for $t \in[\tau, 2 \tau]$.

Similarly, when $t \in[2 \tau, 3 \tau]$, equation (1.5) becomes

$$
\left.z^{\prime}(t)+\lambda k f_{1}(z(t))+M f_{2}\left(z_{2}(t-\tau)\right)\right)=0 .
$$

We denote $z(t)$ as $z_{3}(t)$ for $t \in[2 \tau, 3 \tau]$. This process can be repeated. The obtained equations are ordinary differential equations. It is well known that the convergent order of the linear $\theta$-method for ordinary differential equations is equal $p=1$ for $\theta \neq 1 / 2$ and $p=2$ for $\theta=1 / 2$. So we have

$$
z_{n}-z\left(t_{n}\right)=\mathcal{O}\left(h^{p}\right) .
$$

Hence

$$
x_{n}-x\left(t_{n}\right)=\frac{1}{k}\left(\exp \left(-z_{n}\right)-\exp \left(-z\left(t_{n}\right)\right)\right)=\frac{1}{k} \exp \left(-\delta_{n}\right)\left(z_{n}-z\left(t_{n}\right)\right)=\mathcal{O}\left(h^{p}\right),
$$

where $\delta_{n}$ is in between $z_{n}$ and $z\left(t_{n}\right)$.

In fact, since the linear $\theta$-method is a special form of the Runge-Kutta method, we can easily get (2.6) from [1].

It is obvious that $x_{n}$ is oscillatory about $K^{*}$ if and only if $z_{n}$ is oscillatory. In order to investigate oscillations of (2.2), we only need to study equation (2.1). From [5], it is known that

$$
\left\{\begin{array}{lll}
u f_{1}(u)>0 & \text { for } \quad u \neq 0, & \lim _{u \rightarrow 0} f_{1}(u) / u=1, \\
u f_{2}(u)>0 & \text { for } \quad u \neq 0, & \lim _{u \rightarrow 0} f_{2}(u) / u=1
\end{array}\right.
$$


The linearized equation associated with equation (2.1) is given by

$$
z_{n+1}=z_{n}-h \theta \lambda k z_{n+1}-h \theta M z_{n+1-m}-h(1-\theta) \lambda k z_{n}-h(1-\theta) M z_{n-m},
$$

that is

$$
z_{n+1}-R(-\lambda k h) z_{n}+\frac{M \theta h}{1+\theta \lambda k h} z_{n+1-m}+\frac{M(1-\theta) h}{1+\theta \lambda k h} z_{n-m}=0
$$

where $R(-\lambda k h)=(1-(1-\theta) \lambda k h) /(1+\theta \lambda k h)$ is the stability function of the linear $\theta$-method. It follows from [2] that equation (2.1) oscillates if equation (2.9) oscillates in the case of (2.7).

Definition 3. Equation (2.2) is said to be oscillatory if every solution of (2.2) is oscillatory.

It is natural to ask whether (2.2) is oscillatory when (1.1) oscillates. Until now we only know the sufficient condition $M e^{\lambda k \tau} \tau>1 / e$ for oscillations of equation (1.1). In the following we assume $M e^{\lambda k \tau} \tau>1 / e$ and investigate the conditions under which $(2.2)$ is oscillatory.

In the rest of the paper, we denote $M e^{\lambda k \tau} \tau$ by $a$ for simplicity.

Lemma 2. The characteristic equation of Eq.(2.8) is given by

$$
\xi=R\left(-h\left(\lambda k+M \xi^{-m}\right)\right) \text {. }
$$

Proof. We look at numerical approximations for (2.8) of the form $z_{n}=\xi^{n} z_{0}$, then we get

$$
\begin{aligned}
\xi^{n+1} z_{0}= & \xi^{n} z_{0}-h \theta \lambda k \xi^{n+1} z_{0}-h \theta M \xi^{n+1-m} z_{0} \\
& -h(1-\theta) \lambda k \xi^{n} z_{0}-h(1-\theta) M \xi^{n-m} z_{0} .
\end{aligned}
$$

After simple computations it follows that

$$
\xi=1-h \theta\left(\lambda k+M \xi^{-m}\right) \xi-h(1-\theta)\left(\lambda k+M \xi^{-m}\right),
$$

which leads to the characteristic equation

$$
\xi=\frac{1-h(1-\theta)\left(\lambda k+M \xi^{-m}\right)}{1+h \theta\left(\lambda k+M \xi^{-m}\right)}=1-\frac{h\left(\lambda k+M \xi^{-m}\right)}{1+\theta h\left(\lambda k+M \xi^{-m}\right)} .
$$

Since the stability function of the linear $\theta$-method is $R(x)=\frac{1+(1-\theta) x}{1-\theta x}$, then the characteristic equation of (2.8) is given by (2.10).

Lemma 3. If $a>1 / e$, then the characteristic equation (2.10) has no positive roots for $0 \leq \theta \leq 1 / 2$.

Proof. Let $f(\xi)=\xi-R\left(-h\left(\lambda k+M \xi^{-m}\right)\right)$. It is known from $[13,15]$ that

$$
R\left(-h\left(\lambda k+M \xi^{-m}\right)\right) \leq \exp \left(-h\left(\lambda k+M \xi^{-m}\right)\right), \quad \xi>0, \quad 0 \leq \theta \leq 1 / 2 .
$$


In the following, we will prove $g(\xi)=\xi-\exp \left(-h \lambda k-h M \xi^{-m}\right)>0$ for $\xi>0$. Suppose that there is a $\xi_{0}>0$ such that $g\left(\xi_{0}\right) \leq 0$, then $\xi_{0} \leq \exp (-h \lambda k-$ $\left.h M \xi_{0}^{-m}\right)$, and $\xi_{0}^{m} \leq \exp \left(-\lambda k \tau-M \tau \xi_{0}^{-m}\right)$. Hence

$$
a e=M \tau \exp (\lambda k \tau) e \leq M \tau \xi_{0}^{-m} \exp \left(1-M \tau \xi_{0}^{-m}\right) .
$$

Now we have that

- If $1-M \tau \xi_{0}^{-m}=0$, then $a e \leq 1$, which is a contradiction to $a>1 / e$.

- If $1-M \tau \xi_{0}^{-m} \neq 0$, then since $e^{x}<\frac{1}{1-x}$ for $x<1$ and $x \neq 0$, we again have $a e<1$, which is also a contradiction to $a>1 e$.

Therefore for $\xi>0$

$$
f(\xi)=\xi-R\left(-h\left(\lambda k+M \xi^{-m}\right)\right) \geq \xi-\exp \left(-h \lambda k-h M \xi^{-m}\right)=g(\xi)>0,
$$

which implies that the characteristic equation (2.10) has no positive roots.

In the case of $1 / 2<\theta \leq 1$, we require $m>1$.

Lemma 4. If $a>1 / e$ and $1 / 2<\theta \leq 1$, then the characteristic equation (2.10) has no positive roots for $h<h_{0}$, where

$$
h_{0}=\left\{\begin{array}{l}
\infty, \quad \text { for } \quad M \tau \geq 1, \\
\frac{\tau(1+\lambda k \tau+\ln M \tau)}{1+\lambda k \tau(1-\ln M \tau)}, \quad \text { for } \quad M \tau<1 .
\end{array}\right.
$$

Proof. Since $R\left(-h\left(\lambda k+M \xi^{-m}\right)\right)$ is an increasing function of $\theta$ when $\xi>0$, then for $\xi>0$ and $1 / 2<\theta \leq 1$

$$
R\left(-h\left(\lambda k+M \xi^{-m}\right)\right)=\frac{1-h(1-\theta)\left(\lambda k+M \xi^{-m}\right)}{1+h \theta\left(\lambda k+M \xi^{-m}\right)} \leq \frac{1}{1+h\left(\lambda k+M \xi^{-m}\right)} .
$$

In the following, we will prove that

$$
\xi-\frac{1}{1+h\left(\lambda k+M \xi^{-m}\right)}>0, \quad \xi>0 .
$$

under some conditions. In fact,

$$
\xi-\frac{1}{1+h\left(\lambda k+M \xi^{-m}\right)}=\frac{(1+\lambda k h) \xi^{-m+1}}{1+h\left(\lambda k+M \xi^{-m}\right)} \varphi(\xi),
$$

thus we only need to prove $\varphi(\xi)>0$ for $\xi>0$, where

$$
\varphi(\xi)=\xi^{m}-\frac{1}{1+\lambda k h} \xi^{m-1}+\frac{M h}{1+\lambda k h} .
$$

$\varphi(\xi)$ is the characteristic polynomial of the difference equation

$$
z_{n+1}-z_{n}+\frac{\lambda k h}{1+\lambda k h} z_{n}+\frac{M h}{1+\lambda k h} z_{n+1-m}=0 .
$$


From Theorem 2 and Theorem 3, we have that $\varphi(\xi)$ has no positive roots if and only if

$$
\frac{M h}{1+\lambda k h} \frac{m^{m}}{(m-1)^{m-1}}>\left(1-\frac{\lambda k h}{1+\lambda k h}\right)^{m},
$$

which is equivalent to

$$
\ln M \tau+(m-1) \ln \left(1+\frac{1+\lambda k \tau}{m-1}\right)>0 .
$$

If $M \tau \geq 1$, then (2.12) holds. If $M \tau<1$ and $h<\frac{\tau(1+\lambda k \tau+\ln M \tau)}{1+\lambda k \tau(1-\ln M \tau)}$, then in view of Lemma 1

$$
\ln M \tau+(m-1) \ln \left(1+\frac{1+\lambda k \tau}{m-1}\right)>\ln M \tau+\frac{(m-1)(1+\lambda k \tau)}{m+\lambda k \tau}>0 .
$$

Hence we prove that $\xi-1 /\left(1+h\left(\lambda k+M \xi^{-m}\right)\right)>0, \xi>0$ for $h<h_{0}$, where

$$
h_{0}=\left\{\begin{array}{l}
\infty, \quad \text { for } \quad M \tau \geq 1, \\
\frac{\tau(1+\lambda k \tau+\ln M \tau)}{1+\lambda k \tau(1-\ln M \tau)}, \quad \text { for } \quad M \tau<1
\end{array}\right.
$$

So we have for $h<h_{0}$ and $\xi>0$,

$$
f(\xi)=\xi-R\left(-h\left(\lambda k+M \xi^{-m}\right)\right)>\xi-\frac{1}{1+h\left(\lambda k+M \xi^{-m}\right)}>0
$$

which implies that the characteristic equation (2.10) has no positive roots.

Remark 1. Since $a>\frac{1}{e}$ and $M \tau<1$, then $\frac{\tau(1+\lambda k \tau+\ln M \tau)}{1+\lambda k \tau(1-\ln M \tau)}>0$, hence $h_{0}$ is well defined.

In view of (2.7), Lemma 3 , Lemma 4 and Theorem 2, we have the following theorem.

Theorem 5. If $a>1 / e$, then equation (2.2) is oscillatory for

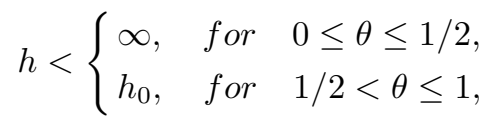

where $h_{0}$ is defined in Lemma 4.

\section{Non-Oscillatory Solutions}

In this section, we will study the asymptotic behaviour of non-oscillatory solutions of (2.2). In [5], there are results about asymptotic behaviour of (1.1).

Lemma 5 [[5]]. Let $x(t)$ be a positive solution of equation (1.1), which does not oscillate about $K^{*}$. Then $\lim _{t \rightarrow \infty} x(t)=K^{*}$. 
Clearly, if Lemma 5 holds, then the solution of (1.5), which does not oscillate, satisfies $\lim _{t \rightarrow \infty} z(t)=0$. In the following, we will prove that $x_{n}$ which is defined by $(2.2)$ can preserve this property.

Lemma 6. Let $z_{n}$ be a solution of equation (2.1), which does not oscillate. Then $\lim _{n \rightarrow \infty} z_{n}=0$.

Proof. Assume that $z_{n}>0$ for $n$ sufficiently large (the proof when $z_{n}<0$ is similar and will be omitted). Then $f_{1}\left(z_{i}\right)>0$ and $f_{2}\left(z_{i}\right)>0$ for $i$ sufficiently large. From (2.1) we have

$$
z_{n+1}-z_{n}+h \theta M f_{2}\left(z_{n+1-m}\right)+h(1-\theta) M f_{2}\left(z_{n-m}\right)<0 .
$$

Similarly, we can get that $z_{n+1}-z_{n}<0$, then $\left\{z_{n}\right\}$ is decreasing. Hence there exists an $\eta \geq 0$ such that

$$
\lim _{n \rightarrow \infty} z_{n}=\eta
$$

We prove that $\eta=0$. Otherwise $\eta>0$, then there is $N \in \mathbb{N}$ and $\varepsilon>0$ such that $0<\eta-\varepsilon<z_{n}<\eta+\varepsilon$ for $n-m>N$, hence $\eta-\varepsilon<z_{n-m}$ and $\eta-\varepsilon<z_{n-m+1}$. So (3.1) yields

$$
z_{n+1}-z_{n}+h \theta M \frac{q_{1}+k^{p}}{p} \frac{e^{p(\eta-\varepsilon)}-1}{q_{1}+k^{p} e^{p(\eta-\varepsilon)}}+h(1-\theta) M \frac{q_{1}+k^{p}}{p} \frac{e^{p(\eta-\varepsilon)}-1}{q_{1}+k^{p} e^{p(\eta-\varepsilon)}}<0,
$$

which implies that $z_{n+1}-z_{n}<B<0$, where $B=h M \frac{q_{1}+k^{p}}{p} \frac{1-e^{p(\eta-\varepsilon)}}{q_{1}+k^{p} e^{p(\eta-\varepsilon)}}$. Thus $z_{n} \rightarrow-\infty$ as $n \rightarrow \infty$, which contradicts to (3.2).

Theorem 6. Let $x_{n}$ be a positive solution of equation (2.2), which does not oscillate about $K^{*}$, then $\lim _{n \rightarrow \infty} x_{n}=K^{*}$.

\section{Numerical Experiments}

In order to give a numerical illustration to the conclusions of this paper, we consider the following equation [6]

$$
x^{\prime}(t)+\frac{\frac{0.0856}{3} x(t) x^{62.62}(t-15)}{\frac{73}{7} 40^{62.62}+x^{62.62}(t-15)}=0.1 .
$$

From [6], we know that equation (4.1) is oscillatory. In Fig. 1, we present the graphs of the analytic solution $x(t)$ and the numerical solution $x_{n}$ respectively. We can see that the numerical solution $x_{n}$ is oscillatory, which is in agreement with Theorem 5 .

We shall use formula (2.2) with different $\theta$ listed in Table 1 with the stepsize $h=\frac{\tau}{m}$ to get the numerical solution at $t=150$, where the true solution is $x(150)=40.689$. In Table 1 , we list the errors of the numerical solution at $t=150$ and the ratio of the errors of the case $m=50$ over that of $m=100$. We can see that the numerical method (2.2) preserves the order of convergence, which is in agreement with the conclusions done in Theorem 4. 


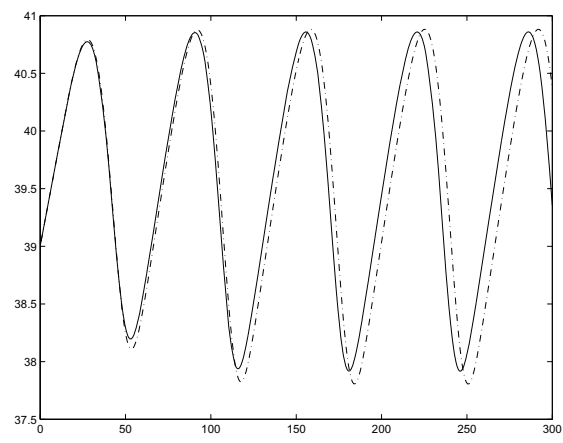

Figure 1. The analytic solution $x(t)$ and the numerical solution $x_{n}$ for Eq.(4.1) with $\theta=0.2$ and $h=0.75$. The solid line denotes $x(t)$ and the dashdot line denotes $x_{n}$.

Table 1. The errors of the exponential linear $\theta$-method for (4.1) at $t=150$.

\begin{tabular}{cccc}
\hline$m$ & $\theta=0.1$ & $\theta=0.5$ & $\theta=0.8$ \\
\hline$m=5$ & $1.278 \mathrm{e}-0$ & $2.402 \mathrm{e}-2$ & $1.583 \mathrm{e}-1$ \\
$m=10$ & $5.370 \mathrm{e}-1$ & $6.387 \mathrm{e}-3$ & $1.218 \mathrm{e}-1$ \\
$m=20$ & $2.311 \mathrm{e}-1$ & $1.621 \mathrm{e}-3$ & $1.024 \mathrm{e}-1$ \\
$m=50$ & $8.133 \mathrm{e}-2$ & $2.604 \mathrm{e}-4$ & $4.952 \mathrm{e}-2$ \\
$m=100$ & $3.860 \mathrm{e}-2$ & $6.513 \mathrm{e}-5$ & $2.607 \mathrm{e}-2$ \\
ratio & 2.107 & 4.000 & 1.900 \\
\hline
\end{tabular}

In the second example we illustrate the validity of Lemma 3 and Lemma 4. From [2], we know that equation (2.8) is oscillatory if and only if the characteristic equation (2.10) has no positive roots. It is known that $f(\xi)$ has positive roots if and only if it has roots in $(0,1)$, which is equivalent to

$$
g(\xi)=\xi^{m+1}-R(-\lambda k h) \xi^{m}+\frac{M \theta h}{1+\theta \lambda k h} \xi+\frac{M(1-\theta) h}{1+\theta \lambda k h}
$$

has roots in $(0,1)$. In order to illustrate oscillations of $(2.8)$ we only need to draw the graphs of $g(\xi)$ in $[0,1]$. We consider the following equation:

$$
x^{\prime}(t)+\frac{0.4 x(t) x(t-2.5)}{3+x(t-2.5)}=3 .
$$

From Theorem 1, equation (4.3) is oscillatory. Let $h=\tau / m$. We draw the graphs of $g(\xi)$ in Fig. 2, where $h_{0}=0.0388$. In (4.3), we have $M \tau=0.1795<1$.

From Fig. 2(a) we can see that the polynomial $g(\xi)$ has two positive roots, $r_{1}=0.86, r_{2}=0.8103$, then (2.8) has a non-oscillatory solution. In (b) and (c) of Fig. 2, we change the value of $\theta$ and $h$ respectively, then we can see the that polynomial $g(\xi)$ has no positive roots. Then equation (2.8) is oscillatory, which is in agreement with Lemma 3 and Lemma 4. In Fig. 2(d), we can see that despite $h>h_{0}$, equation (2.8) is still oscillatory. Then $h_{0}$ in Lemma 4 is not optimal. 


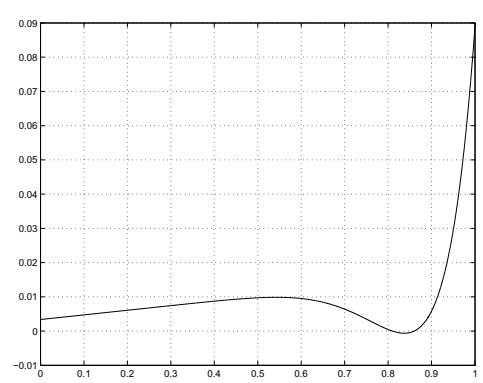

(a) $\theta=0.8, h=0.25$

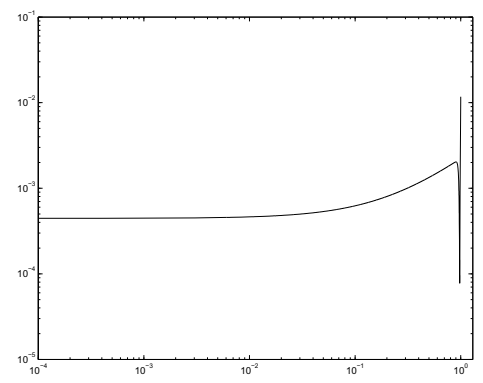

(c) $\quad \theta=0.8, h=0.0313$

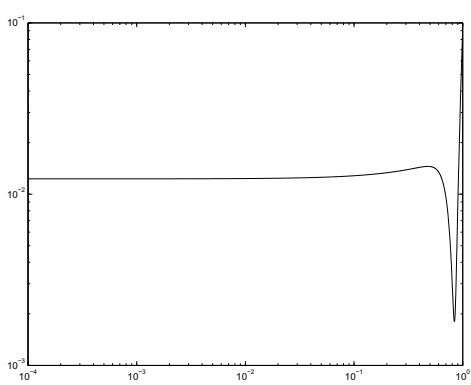

(b) $\theta=0.3, h=0.25$

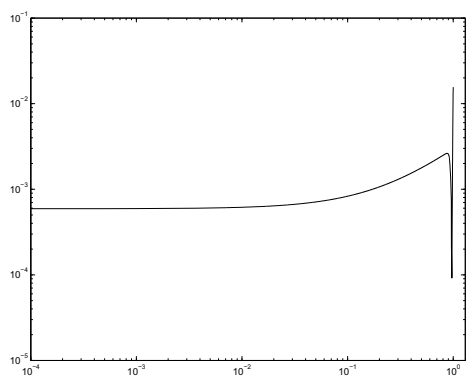

(d) $\theta=0.8, h=0.0417$

Figure 2. The graphs of $g(\xi)$ for equation (4.3).

\section{Conclusions}

In the paper, we investigate oscillations of the numerical solution of a nonlinear delay differential equation of population dynamics. The convergent exponential linear $\theta$-method is constructed. We get conditions under which the numerical solution oscillates in the case of oscillations of the analytic solution. We also prove that non-oscillatory numerical solutions can preserve properties of nonoscillatory analytic solutions. For the future work, we will investigate the approximation more accurately. Moreover, parameter $h_{0}$ in Lemma 4 is not optimal. We will look for another stepsize $h_{1}$, which is more accurate than $h_{0}$ and is easily verified.

\section{Acknowledgements}

The authors are grateful to the referees for the careful reading of the paper and several useful suggestions.

\section{References}

[1] G. Wanner E. Hairer, S.P. Nørsett. Solving Ordinary Differential Equations I: Nonstiff Problems. Springer-Verlag, Berlin Heidelberg, 1993. 
[2] I. Györi and G. Ladas. Oscillation Theory of Delay Differential Equations with Applications. Academic Press, Oxford, 1991.

[3] J.K. Hale. Theory of Functional Differential Equations. Springer-Verlag, New York, 1977.

[4] Q. Kong. Oscillation and asymptotic behavior of a discrete logistic model. Rocky Mount. J. Math., 25:339-349, 1995. Doi:10.1216/rmjm/1181072287.

[5] I. Kubiaczyk and S.H. Saker. Oscillation and stability in nonlinear delay differential equations of population dynamics. Math. Comput. Model., 35:295-301, 2002. Doi:10.1016/S0895-7177(01)00166-2.

[6] P.S. Landa. Nonlinear Oscillations and Waves in Dynamical Systems. Kluwer Academic Publishers, London, 1996.

[7] H.Y. Liang, Q.L. Li and Z.G. Zhang. New oscillatory criteria for higherorder nonlinear neutral delay differential equation. Nonlinear Analysis TMA., 69:1719-1731, 2008. Doi:10.1016/j.na.2007.07.018.

[8] M.Z. Liu, J.F. Gao and Z.W. Yang. Oscillation analysis of numerical solution in the $\theta$-methods for equation $x^{\prime}(t)+a x(t)+a_{1} x([t-1])=0$. Appl. Math. Comput., 186:566-578, 2007. Doi:10.1016/j.amc.2006.07.119.

[9] M.Z. Liu, Jianfang Gao and Z.W. Yang. Preservation of oscillations of the rungekutta method for equation $x^{\prime}(t)+a x(t)+a_{1} x([t-1])=0$. Comput. Math. Appl., 58:1113-1125, 2009. Doi:10.1016/j.camwa.2009.07.030.

[10] M.C. Mackey and L. Glass. Oscillation and chaos in physiological control system. Science, 197:287-289, 1977. Doi:10.1126/science.267326.

[11] D.H. Peng, M.A. Han and H.Y. Wang. Linearized oscillations of first-order nonlinear neutral delay difference equations. Comput. Math. Appl., 45:17851796, 2003. Doi:10.1016/S0898-1221(03)90000-9.

[12] S.H. Saker. Oscillation of second-order nonlinear neutral delay dynamic equations on time scales. J. Comput. Appl. Math., 187:123-141, 2006. Doi:10.1016/j.cam.2005.03.039.

[13] M.H. Song, Z.W. Yang and M.Z. Liu. Stability of $\theta$-methods for advanced differential equations with piecewise continuous arguments. Comput. Math. Appl., 49:1295-1301, 2005. Doi:10.1016/j.camwa.2005.02.002.

[14] Y.G. Sun and S.H. Saker. Oscillation for second-order nonlinear neutral delay difference equations. Appl. Math. Comput., 163:909-918, 2005. Doi:10.1016/j.amc.2004.04.017.

[15] Z.W. Yang, M.Z. Liu and M.H. Song. Stability of Runge-Kutta methods in the numerical solution of equation $u^{\prime}(t)=a u(t)+a_{0} u([t])+a_{1} u([t-1])$. Appl. Math. Comput., 162:37-50, 2005. Doi:10.1016/j.amc.2003.12.081.

[16] A. Zaghrout, A. Ammar and M.M.A. El-Sheikh. Oscillation and global attractivity in delay equation of population dynamics. Appl. Math. Comput., 77:195-204, 1996. Doi:10.1016/S0096-3003(95)00213-8. 